\title{
The MV Model of the Color Glass Condensate for a Finite Number of Sources Including Coulomb Interactions
}

\author{
Larry McLerran ${ }^{\mathrm{a}, \mathrm{c}}$, Vladimir V. Skokov ${ }^{\mathrm{b}}$ \\ ${ }^{a}$ Department of Physics, Brookhaven National Laboratory, Upton, NY 11973 \\ ${ }^{b}$ RIKEN/BNL, Brookhaven National Laboratory, Upton, NY 11973 \\ ${ }^{c}$ Physics Dept, China Central Normal University, Wuhan, China
}

\begin{abstract}
We modify the McLerran-Venugopalan model to include only a finite number of sources of color charge. In the effective action for such a system of a finite number of sources, there is a point-like interaction and a Coulombic interaction. The point interaction generates the standard fluctuation term in the McLerran-Venugopalan model. The Coulomb interaction generates the charge screening originating from well known evolution in $x$. Such a model may be useful for computing angular harmonics of flow measured in high energy hadron collisions for small systems. In this paper we provide a basic formulation of the problem on a lattice.
\end{abstract}

\section{Introduction}

The McLerran-Venugopalan (MV) model of the Color Glass Condensate (CGC) has provided a useful phenomenology of high energy hadronic processes [1, 2]. This model is however lacking in one essential aspect: There is no color charge screening. This lack of color screening follows from the form of the charge density correlation function

$$
\left\langle\rho^{a}\left(y_{1}, \vec{x}_{1}\right) \rho^{b}\left(y_{2}, \vec{x}_{2}\right)\right\rangle=\frac{d \mu^{2}}{d y} \delta^{a b} \delta\left(y_{1}-y_{2}\right) \delta^{(2)}\left(\vec{x}_{1}-\vec{x}_{2}\right)
$$

In this equation, we take the charge densities to be local both in rapidity and in two dimensional transverse coordinate. The locality in rapidity is required for what we wish to do in the following analysis. Upon integrating over the coordinates $y_{1}, \vec{x}_{1}$, we see that 
we get a non-vanishing contribution so that the total charge is non zero. In the MV model, color charge screening is usually argued to occur on a scale size of order the confinement size scale and is outside the range of applicability of the model.

On the other hand, if one evolves the MV model [3, 4, 5], one restores charge neutrality on a scale of order the saturation momentum [6]. The effect of evolution is to modify the charge-charge correlation function, and is best understood in transverse momentum space:

$$
\left\langle\rho^{a}\left(y_{1}, \vec{k}_{1}\right) \rho^{b}\left(y_{2}, \vec{k}_{2}\right)\right\rangle=(2 \pi)^{2} \frac{d \mu^{2}}{d y} \delta^{a b} \delta\left(y_{1}-y_{2}\right) \delta^{(2)}\left(\vec{k}_{1}+\vec{k}_{2}\right) \Delta\left(\vec{k}_{1}\right) .
$$

The total charge in a transverse plane corresponding to a fixed rapidity is the limit as $k \rightarrow 0$ of the Fourier transformed charge density. The requirement of charge neutrality is that

$$
\lim _{k \rightarrow 0} \Delta(k) \rightarrow 0
$$

It should be noted here that we are not being careful about gauge invariance when discussing a charge charge correlation function at non-zero spatial separation. This is fixed up by inserting line ordered phases attached to the charge densities. This complication comes in when one goes beyond leading order in coupling in the MV model; in this case fluctuations in the transverse vector potential become important.

In a recent paper, we wrote down a phenomenological model which includes corrections to the MV that enforce charge neutrality [7]. This model has

$$
\begin{aligned}
\Delta(k) & =\frac{k^{2}}{k^{2}+m^{2}} \\
& =1-\frac{m^{2}}{k^{2}+m^{2}} .
\end{aligned}
$$

Here $m$ is of order the saturation momentum. The charge induced by the first term on the RHS of this equation is precisely cancelled by the second term. Note that in coordinate space, the second term Fourier transforms to a $K_{0}(m r)$ so that the neutralizing polarization charge density is exponentially well localized around the initial charge density. 
One effect of including the polarization charge density is to generate angular moments of elliptic flow for radiation from the CGC [7]. Without final state interactions, one generates even moments for multiparticle correlations which at high momentum have the correct semiquantitative features to describe flow harmonics seen in $p p$ and $p A$ collisions. However these correlations are only present if we further generalize our model to a finite number of emission sources. As happened for ellipticities in Glauber or CGC computations $[8,9]$, fluctuation induced ellipticities vanish for large numbers of emitters. It also should be noted that the odd flow moments vanish and may require some degree of final state interaction and a deeper understanding of the nuclear wave function. In any case, whether these flow contributions are essentially modified by final state interactions or not, it is important to understand the initial state contribution to such moments.

It is the purpose of this paper to properly motivate a theory of a finite number of color charge emitters. We shall see that the $m^{2}$ correction to the charge density correlation function arise from an effective Coulomb interaction. This Coulomb interaction might be viewed as arising from a higher order term in the operator product expansion for

the effective theory of sources in the McLerran-Venugopalan model. It does not directly arise from Coulomb interactions of the QCD Lagrangian from which this theory is derived. Although the interpretation of this term within our effective action is amusing, its inclusion is strictly to enforce the charge neutrality constraints associated with evolution in $x$.

\section{The Coulomb Interaction and Charge Screening}

We begin by observing that the action that generates the screened propagator above is

$$
S=\int d y \int \frac{d^{2} k_{\perp}}{(2 \pi)^{2}} \rho\left(y, \vec{k}_{\perp}\right)\left[\frac{1}{2 \bar{\mu}^{2}}\left(1+\kappa \frac{\bar{\mu}^{2}}{k_{\perp}^{2}}\right)\right] \rho\left(y,-\vec{k}_{\perp}\right)
$$

where

$$
\bar{\mu}^{2}=\frac{d \mu^{2}}{d y}
$$


is the two dimensional charge density per unit rapidity. And we introduced the dimensionless coefficient $\kappa=m^{2} / \bar{\mu}^{2}$. Note that the second term in this equation is simply the two-spatial dimensional Coulomb interaction which in coordinate space is

$$
S_{C}=\int d y \int d^{2} x_{\perp} d^{2} y_{\perp} \rho\left(y, \vec{x}_{\perp}\right)\left[\frac{\kappa}{8 \pi} \ln \frac{1}{\left(\vec{x}_{\perp}-\vec{y}_{\perp}\right)^{2} \Lambda_{\mathrm{IR}}^{2}}\right] \rho\left(y, \vec{y}_{\perp}\right) .
$$

The dependence upon the IR scale arises from regulating the integration over small $k_{\perp}$ in the Fourier transformed action integration. Note that if it is much larger than all scales in the problem, it projects the total color charge in a unit of rapidity onto zero. Alternatively, if the total color charge in a unit of rapidity is zero, which we will assume in what follows, then there is no dependence upon the IR scale, which may therefore be replaced by some convenient physical scale size of order the size of the system being considered.

The parameter $\bar{\mu}^{2}=d \mu^{2} / d y$ is proportional to the charge squared per unit area per unit rapidity, and is therefore proportional to the saturation momentum. We can see this explicitly for adjoint representation sources (see e.g. $[6,7]$ ), where

$$
\mu^{2}=4 \pi \alpha_{s} N_{c} N / S_{\perp}
$$

Here $N$ is the total number of particle integrated over rapidity, and $S_{\perp}$ is the transverse area. The saturation momentum in the MV model up to logarithmic corrections (see e.g. [6]) is

$$
Q_{s}^{2}=\alpha_{s} N_{c} \mu^{2}
$$

Note that for a truly two dimensional Coulomb interaction there is a dimensional scale associated with the two dimensional coupling. This has disappeared from this formula, and has been replaced by the dimensionless three spatial dimensional coupling. We can think of our effective action as being associated with a dimensional scale of order the saturation momentum, which is then weighed in the action like $e^{-E / T}$ where the effective temperature $T$ is proportional to the saturation momentum. 
The Coulomb 2-dimensional interaction is natural to expect when we have Lorentz boosted Coulomb fields. The electric field around a point charge is boosted to

$$
F^{i+} \propto \delta\left(x^{-}\right) \frac{\hat{r}}{r}
$$

and arises from a vector potential $A^{+} \propto \ln (r)$. This vector potential has an infrared cutoff originating from a vector potential of the form

$$
A^{+} \propto \frac{\gamma}{\sqrt{\gamma^{2} x^{-2}+x_{T}^{2}}}
$$

In the limit $\gamma \rightarrow \infty$, this reduces to the logarithmic potential, so long as $r \gg x^{-} / \gamma$. This explains the infrared cutoff in the two dimensional potential. The origin of locality in rapidity follows because $x^{-} \sim 1 / \gamma$ implies that $\Delta x^{-}=\tau_{1} e_{1}^{y}-\tau_{2} e_{2}^{y} \sim 1 / \gamma$ or $\Delta y=\ln \left(\tau_{1} / \tau_{2}\right)$. This means interactions are localized within about a unit of rapidity.

Although it might appear that the Coulomb interaction under investigation might originate from a simple interpretation of the underlying gauge theory, it is not the case, because $S_{C}$ is not of the covariant form $F^{i+} F^{i-}$. It should be rather thought of as arising from an operator product expansion for the effective action of the sources.

\section{Lattice formulation for the effective action and numerical results}

This theory can be put on a finite grid. There is a mild logarithmic singularity at short distance in the Coulomb potential can be regulated by the finite grid size in the transverse direction. We can choose the spacing to be one unit of rapidity, and this is a good approximation since distributions are slowly varying over one unit of rapidity, and the Coulomb interaction, for the reasons discussed above should have a range of about one unit of rapidity if the interaction in rapidity is properly accounted for. This is of course finite in the limit the grid size shrinks to zero. We also assume the IR divergence is taken care of by requiring total color charge neutrality on a confining scale.

It is useful to think about this theory for a discrete number of emitters, that is we imagine that the sources of the color charge are single gluons carrying an amount of charge 
corresponding to the octet representation of the gauge group. This pushes the classical description of the color charge to its limit of validity. To have a truly proper theory one should treat the color charge operator as a matrix and solve the theory like was done in static matrix models. For our proposes, we will however treat the color charge as a continuous classical variable. Our interest is in understanding the effects of having a finite number of particles and writing down a theory that corresponds to such a finite number of sources. Presumably, if we discretize on a small scale, but consider quantities whose scale of variation is over a size scale that includes many units of color charge, treating the color sources as classical variable should be well behaved.

Before the discretization is performed, it is necessary to regularize the interaction and maintain positivity of the action. The last requirement does not allow one to use a naive delta-function point-like source on the lattice. We can maintain the positivity of the action when we spread the sources over the region of size $\Lambda_{\mathrm{UV}}^{-1}$, i.e. in the momentum space we consider

$$
\rho^{a}\left(y, \vec{k}_{\perp}\right)=\sum_{i} \frac{\Lambda_{\mathrm{UV}}^{2}}{k^{2}+\Lambda_{\mathrm{UV}}^{2}} \xi_{i}^{a}(y) \exp \left(-i \vec{k}_{\perp} \vec{x}_{i}\right)
$$

or transforming into the coordinate space

$$
\rho^{a}\left(y, \vec{x}_{\perp}\right)=\frac{1}{2 \pi} \sum_{i} \xi_{i}^{a}(y) K_{0}\left(\left|\vec{x}_{\perp}-\vec{x}_{i}\right|\right) \Lambda_{\mathrm{UV}}
$$

Note that in the limit of large $\Lambda_{\mathrm{UV}}$ Eq. (12) reduces to the Fourier transform of the delta-function.

Thus, combining Eq. (13) and Eq. (5) results in the MV action which is manifestly positive

$$
S_{\mathrm{MV}}[\{\xi\},\{\vec{x}\}]=\int d y \sum_{i, j} \xi_{i}^{a}(y)\left[\frac{\left|\hat{\vec{x}}_{i}-\hat{\vec{x}}_{j}\right| K_{1}\left(\left|\hat{\vec{x}}_{i}-\hat{\vec{x}}_{j}\right|\right)}{8 \pi \hat{\mu}^{2}}\right] \xi_{j}^{a}(y),
$$

where in order to simplify the notations we introduced the following dimensionless vari- 
ables $\hat{x}=x \Lambda_{\mathrm{UV}}$ and $\hat{\mu}=\bar{\mu} / \Lambda_{\mathrm{UV}}$. The Coulomb term is then

$$
\begin{aligned}
& S_{C}[\{\xi\},\{\vec{x}\}]=\int d y \int d^{2} x_{\perp} d^{2} y_{\perp} \rho\left(y, \vec{x}_{\perp}\right)\left[\frac{\kappa}{4 \pi} \ln \frac{1}{\left|\vec{x}_{\perp}-\vec{y}_{\perp}\right| \Lambda_{\mathrm{IR}}}\right] \rho\left(x_{-}, \vec{y}_{\perp}\right)= \\
& \int d y \sum_{i, j} \xi_{i}^{a}(y)\left[\frac{\kappa}{4 \pi}\left(\ln \frac{1}{\left|\hat{\vec{x}}_{i}-\hat{\vec{x}}_{j}\right| \hat{\Lambda}_{\mathrm{IR}}}-K_{0}\left(\left|\hat{\vec{x}}_{i}-\hat{\vec{x}}_{j}\right|\right)-\frac{1}{2}\left|\hat{\vec{x}}_{i}-\hat{\vec{x}}_{j}\right| K_{1}\left(\left|\hat{\vec{x}}_{i}-\hat{\vec{x}}_{j}\right|\right)\right)\right] \xi_{j}^{a}(y) .
\end{aligned}
$$

We note that the kernel enclosed in the rectangular brackets is finite at small distances due to compensation of the logarithmic singularities in the first and second terms. We will return to this point in the next section.

Now to perform the simulations, one can use a spatial rectangular lattice with the lattice spacing $a$ and transverse length $L$. The rapidity is to be discretized as well to simulate a finite extent of sources in the rapidity direction. This means that the integrals with respect to rapidity are replaced by sums as follows

$$
\int d y \rightarrow \sum_{n} \delta y
$$

It is interesting that the action for a finite number of sources is similar to that for the MV model except that in the MV model, sources are allowed to fluctuate in all of the two dimensional space. The typical charge per grid unit in the MV model is the same as the typical charge per particle in our discretized model. The essential difference is that for our discretized model, only a finite fraction of the transverse area is covered by sources. This is what ultimately will allow for generation of elliptical flow moments in radiation from the grid, for effect that vanish in the limit that the number of sources $N \rightarrow \infty$.

As a test of our formalism, we will compute the source-source correlator and the $\mathrm{S}$ matrix for single particle scattering by numerically solving the finite $N$ discretized version of our theory.

Consider $N$ sources distributed in the target. We will assume that the distribution is uniform in rapidity and thus the number of sources per a rapidity slice is given by $N / N_{y}$ where $N_{y}$ is the number of rapidity slices. 
The sources are spread as we discussed before. Their (non-Abelian) WeizsäckerWilliams fields are in covariant gauge,

$$
A^{+a}\left(y, \vec{x}_{\perp}\right)=-\frac{g}{\nabla_{\perp}^{2}} \rho^{a}\left(y, \vec{x}_{\perp}\right) .
$$

The only non-vanishing field strength is $F^{+i}=-\partial^{i} A^{+}$. The (light-cone) electric field is

$$
E^{i}=\int d x^{-} F^{+i}=-\partial^{i} \int d x^{-} A^{+} .
$$

The propagation of a fast charge in this field is described by an eikonal phase given by a light-like Wilson line $V\left(\vec{x}_{\perp}\right)$ :

$$
V\left(\vec{x}_{\perp}\right)=\mathbb{P} \exp \left\{-i g \int d y A\left(y, \vec{x}_{\perp}\right)\right\},
$$

where $\mathbb{P}$ denotes path-ordering in rapidity $y$ and the field can be found by solving Eq. (17)

$$
A\left(y, \vec{x}_{\perp}\right)=\frac{g}{2 \pi} \sum_{i} \xi_{i}(y)\left(\ln \left[\frac{1}{\left|\hat{\vec{x}}_{\perp}-\hat{\vec{x}}_{i}\right| \hat{\Lambda}_{\mathrm{IR}}}\right]-K_{0}\left(\left|\hat{\vec{x}}_{\perp}-\hat{\vec{x}}_{i}\right|\right)\right) .
$$

Here $\hat{\Lambda}_{\mathrm{IR}}=\Lambda_{\mathrm{IR}} / \Lambda_{U V}$. Note that $A\left(y, \vec{x}_{\perp}\right)$ is finite at any $\vec{x}_{\perp}$ except for infinite distances owing to the following identity for small $\hat{r}$

$$
\ln \left[\frac{1}{\hat{r} \hat{\Lambda}_{\mathrm{IR}}}\right]-K_{0}(\hat{r})=\gamma+\log \left(\frac{1}{2 \hat{\Lambda}_{\mathrm{IR}}}\right)+\frac{1}{4} \hat{r}^{2}\left(\log \left(\frac{\hat{r}}{2 e}\right)+\gamma\right)+\mathcal{O}\left(x^{3}\right) .
$$

The S-matrix for scattering of this charge off the given target field configuration is

$$
\mathcal{S}_{\rho}\left(\vec{r}_{\perp}, \vec{b}_{\perp}\right) \equiv \frac{1}{d_{R}} \operatorname{tr} V^{\dagger}\left(\vec{x}_{\perp}\right) V\left(\vec{y}_{\perp}\right), \quad \vec{r}_{\perp} \equiv \vec{x}_{\perp}-\vec{y}_{\perp}, \quad 2 \vec{b}_{\perp} \equiv \vec{x}_{\perp}+\vec{y}_{\perp} .
$$

In momentum space, this can be written in the following form

$$
\mathcal{S}_{\rho}\left(\vec{k}_{\perp}\right)=\frac{1}{d_{R}} \operatorname{tr}\left[\int d^{2} x_{\perp} d^{2} y_{\perp} e^{i \vec{k}_{\perp}\left(\vec{x}_{\perp}-\vec{y}_{\perp}\right)} V^{\dagger}\left(\vec{x}_{\perp}\right) V\left(\vec{y}_{\perp}\right)\right]=\frac{1}{d_{R}} \operatorname{tr} V^{\dagger}\left(\vec{k}_{\perp}\right) V\left(\vec{k}_{\perp}\right),
$$

The S-matrix is obtained by averaging $\mathcal{S}_{\rho}\left(\vec{k}_{\perp}\right)$ with respect to target configurations. Those are to be generated using the effective action we defined above. Here it is important to realize that the effective action is quadratic in color charges $\xi_{i}$, and thus they can be 


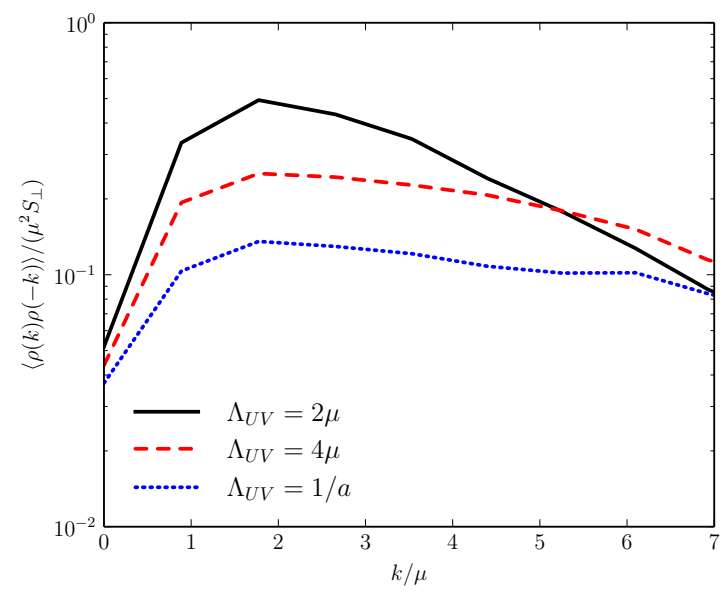

Figure 1: The correlator $\langle\rho(\vec{k}) \rho(-\vec{k})\rangle$ normalized to yield 1 in the limit of the infinite number of sources. The number of sources is $N=500$ and $\kappa=1$.

integrated out, leaving us with a non-trivial complicated function of the positions of the charges. We prefer not to follow this way but rather to simulate the configurations using the Metropolis Monte-Carlo algorithm as detailed in Appendix.

Lets first consider the source-source correlator for different number of particles $n$ per rapidity slice. We check that there is a convergence if large values of $\Lambda_{\mathrm{UV}}$ are considered. From very general considerations, it is clear that if the momentum $k_{\perp}$ is larger than $\Lambda_{\mathrm{UV}}$ model results will be sensitive to the details of the source regularization in Eq. (12). Indeed Fig. 1 for $\Lambda_{\mathrm{UV}}=2 \mu$ shows a very significant variation in the correlator $\langle\rho(\vec{k}) \rho(-\vec{k})\rangle$ which, at large $k_{\perp}$, is expected to be independent of the momentum and assume $\mu^{2} S_{\perp}$ in the limit of the infinite number of sources. Nonetheless independent of the scale $\Lambda_{\mathrm{UV}}$ we see the color neutralization driving the value of the correlator to zero at small momentum.

In our calculations we prefer to set the scale $\Lambda_{\mathrm{UV}}$ to the largest possible on the lattice $1 / a$. In this case, the $\rho \rho$ correlator is approximately constant above the color neutralization scale.

The dependence of the correlator on the number of sources is displayed in Fig. 2 for $\kappa=0$ and 1 . The offset of the Debye screening can be seen for the number of particles $N=100$. 


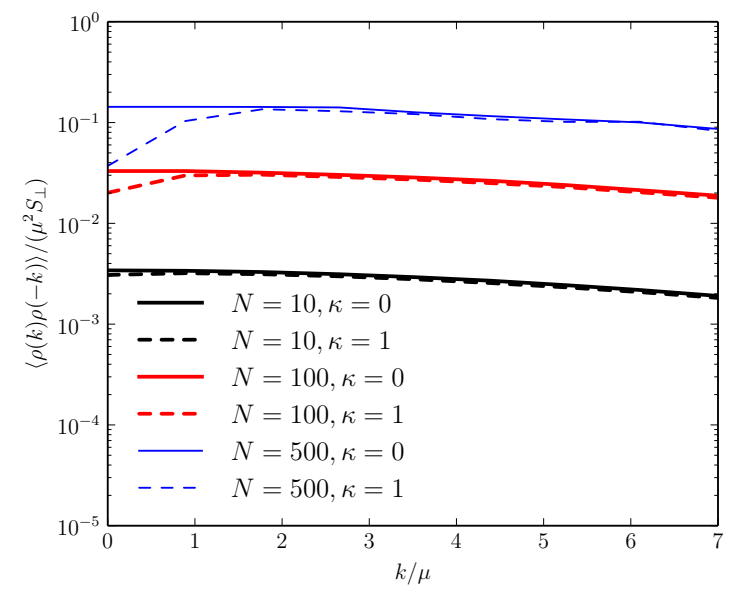

Figure 2: The correlator $\langle\rho(\vec{k}) \rho(-\vec{k})\rangle$ normalized to yield 1 in the limit of the infinite number of sources. The solid (dashed) lines correspond to MV model without (with) Coulomb interaction. The infrared cutoff is $\Lambda_{\mathrm{IR}}=1 / L$, where $L$ is the system size.

To check that the model reproduces $1 / k_{\perp}^{4}$ dependence of the particle spectrum, we computed the S matrix, depicted in Fig. 3. As expected, the results are not very sensitive to the Coulomb interaction. This however should not be discouraging since the effect Coulomb interaction is essential for the two particle correlation function, as discussed in Ref. [7].

\section{Conclusions}

We considered an extension of the McLerran-Venugopalan to include a finite number of sources of color charge and Coulomb interactions between color charges. To simulate this theory on the lattice, the sources are regularized in a manner that maintains the positivity of the MV action. We showed that if the corresponding scale of the transverse momentum is large it leads to the expected results of approximately constant $\rho \rho$-correlator. We investigated the dependence on $\kappa$ and showed the emergence of the color neutralization in the model. In this paper, we did not consider the two particle correlation function, this is the ultimate aim of the model which will be reported elsewhere.

It is useful to put this work in the context of previous work that has been done. In 


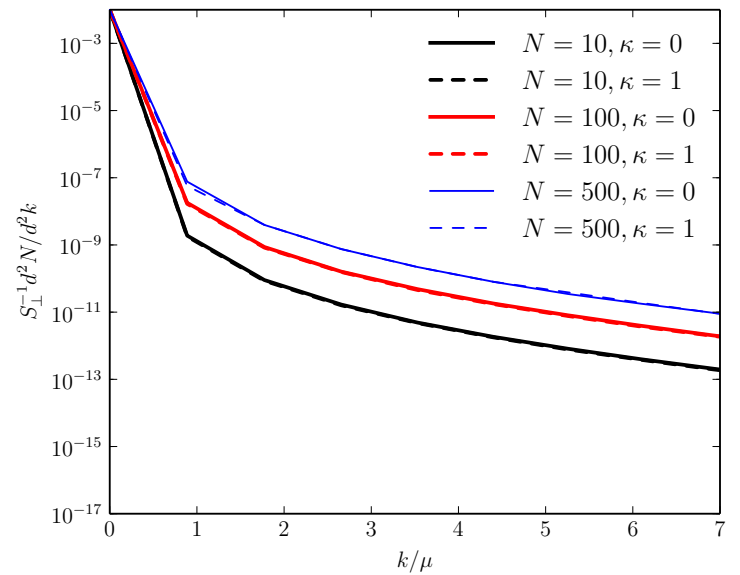

Figure 3: The differential spectrum $d^{2} N / d^{2} k$ normalized by the transverse area of the target. The solid (dashed) lines corresponds to MV model without (with) Coulomb interaction. The ultraviolet and infrared cutoffs are set to $\Lambda_{\mathrm{UV}}=4 \mu$ and $\Lambda_{\mathrm{IR}}=1 / L$, where $L$ is the system size.

early work on flow harmonics, it was shown that a finite number of nucleon sources can generate the observed structure seen in nuclear collisions [10,11]. More recently it was shown that for a finite sources in $p A$ collisions can semi-quantitatively reproduce flow harmonics $[8,9]$. The even flow harmonic in $p A$ and $p p$ collisions might also be described by intrinsic correlations of gluon emitted from a Glasma'[12, 13], an effect that survives for an infinite number of sources but is non-leading in the number of colors. It was then suggested that such effects might arise from scattering off of finite size domains of colored charge $[14,15]$. Such flow harmonics arise in computations using the evolution of classical fields $[16,17,18]$. The purpose of this paper was to expand upon the idea that the origin of such color fluctuations is in the finite number of color sources [7], and to propose an explicit theory that allows for the computation of these effects, properly including the effects of color screening.

The computation of multi-particle correlations is most likely a numerically intensive problem. But one needs to first formulate a well defined theoretical framework in which these computations are embedded. The goal of this paper was to make such a formulation. The numerical results presented in this paper are only to show that this framework has 
no obvious flaw. A computation of two and higher particle correlations is quite difficult, although the numerical Monte Carlo methods advocated in this paper may provide computations of low order correlation functions. With a well defined path integral formulation of the problem, perhaps analytic results might be envisaged. The problem as formulated is a very simple one: The classical partition function for a gas of a finite number of particles interacting with point like and Coulombic interactions.

\section{Acknowledgements}

The authors gratefully acknowledge the comments of Jean Paul Blaizot, Soren Schlichting, Bjoern Schenke, Raju Venugopalan and Ulrich Heinz.

The authors are supported under Department of Energy contract number Contract No. DE-SC0012704.

\section{Appendix A: Numerical procedure}

The averaging with respect to the target configurations must be performed with the weight function

$$
W[\{x\},\{\xi\}]=\exp (-S(\{x\},\{\xi\})) .
$$

In order to generate the configurations we use the Metropolis algorithm for each rapidity slice. Although the functional $S(\{x\},\{\xi\})$ is quadratic in $\{\xi\}$, it has a very non-trivial structure in the sources' positions $\{x\}$. The Metropolis algorithm is performed along the following steps:

1. Initially $N / N_{y}$ sources are randomly distributed on a two-dimensional rectangular grid with the spatial extent $L$ and the spacing $a . N_{y}$ is the number of the rapidity slices, see below. For each point, the initial color vector $\xi^{a}$ is generated according to the Gaussian probability distribution with zero mean and the variance $\bar{\mu}$.

2. The Metropolis step is performed. One source is randomly chosen; a trial change, $\Delta S$, of the action $S=S_{\mathrm{MV}}+S_{C}$ is computed; if the source position is randomly 
adjusted by a step $a$ in a randomly chosen direction and its color vector receive a random contribution distributed according to a Gaussian with zero mean and the variance of $10 \%$ of $\bar{\mu}$.

3. If $\Delta S<0$, the change of the position and the color vector is accepted. Otherwise the change is accepted with the probability $\exp (-S)$.

4. The steps 2 and 3 are repeated until equilibrium is reached.

5. The configurations are collected. To avoid autocorrelation, there is at least $10 \mathrm{MC}$ sweeps between each saved configuration.

After the configurations are collected, we compute the source density $\rho\left(y, \vec{x}_{\perp}\right)$ and the corresponding $A\left(y, \vec{x}_{\perp}\right)$. The number of the rapidity slices is finite in our approach and each rapidity slice has approximately one unit in rapidity $\delta y=1$. We assume that the sources are generated in each rapidity slice independently. Overall we consider $N_{y}$ rapidity slices, we fix $N_{y}=5$. Owing to the path ordering the Wilson line is given by the product of exponentials computed in each rapidity slice

$$
V\left(\vec{x}_{\perp}\right)=\prod_{i=1}^{N_{y}} \exp \left(-i g A\left(y_{i}, \vec{x}_{\perp}\right)\right), y_{i}=i \delta y .
$$

By performing Fourier transformation and averaging with respect to the target configurations we can extract $S\left(\vec{k}_{\perp}\right)$.

\section{References}

[1] L. D. McLerran and R. Venugopalan, Phys. Rev. D 49 (1994) 2233 doi:10.1103/PhysRevD.49.2233 [hep-ph/9309289].

[2] L. D. McLerran and R. Venugopalan, Phys. Rev. D 49 (1994) 3352 doi:10.1103/PhysRevD.49.3352 [hep-ph/9311205].

[3] E. Iancu, A. Leonidov and L. D. McLerran, Nucl. Phys. A 692 (2001) 583 doi:10.1016/S0375-9474(01)00642-X [hep-ph/0011241]. 
[4] E. Ferreiro, E. Iancu, A. Leonidov and L. McLerran, Nucl. Phys. A 703 (2002) 489 doi:10.1016/S0375-9474(01)01329-X [hep-ph/0109115].

[5] A. H. Mueller, Nucl. Phys. B 643, 501 (2002) doi:10.1016/S0550-3213(02)00745-9 [hep-ph/0206216].

[6] E. Iancu, K. Itakura and L. McLerran, Nucl. Phys. A 708 (2002) 327 doi:10.1016/S0375-9474(02)01010-2 [hep-ph/0203137].

[7] L. McLerran and V. Skokov, arXiv:1510.08072 [hep-ph].

[8] A. Bzdak, P. Bozek and L. McLerran, Nucl. Phys. A 927 (2014) 15 doi:10.1016/j.nuclphysa.2014.03.007 [arXiv:1311.7325 [hep-ph]].

[9] L. Yan, J. Y. Ollitrault and A. M. Poskanzer, Phys. Rev. C 90 (2014) 2, 024903 doi:10.1103/PhysRevC.90.024903 [arXiv:1405.6595 [nucl-th]].

[10] R. Andrade, F. Grassi, Y. Hama, T. Kodama and O. Socolowski, Jr., Phys. Rev. Lett. 97 (2006) 202302 doi:10.1103/PhysRevLett.97.202302 [nucl-th/0608067].

[11] B. Alver and G. Roland, Phys. Rev. C 81 (2010) 054905 Erratum: [Phys. Rev. C 82 (2010) 039903] doi:10.1103/PhysRevC.82.039903, 10.1103/PhysRevC.81.054905 [arXiv:1003.0194 [nucl-th]].

[12] A. Dumitru, K. Dusling, F. Gelis, J. Jalilian-Marian, T. Lappi and R. Venugopalan, Phys. Lett. B 697 (2011) 21 doi:10.1016/j.physletb.2011.01.024 [arXiv:1009.5295 [hep-ph]].

[13] K. Dusling and R. Venugopalan, Phys. Rev. D 87 (2013) no.5, 054014 doi:10.1103/PhysRevD.87.054014 [arXiv:1211.3701 [hep-ph]].

[14] A. Dumitru and A. V. Giannini, Nucl. Phys. A 933, 212 (2015) doi:10.1016/j.nuclphysa.2014.10.037 [arXiv:1406.5781 [hep-ph]]. 
[15] A. Dumitru, L. McLerran and V. Skokov, Phys. Lett. B 743, 134 (2015) doi:10.1016/j.physletb.2015.02.046 [arXiv:1410.4844 [hep-ph]].

[16] A. Krasnitz, Y. Nara and R. Venugopalan, Nucl. Phys. A 717, 268 (2003) doi:10.1016/S0375-9474(03)00636-5 [hep-ph/0209269].

[17] T. Lappi, B. Schenke, S. Schlichting and R. Venugopalan, JHEP 1601, 061 (2016) doi:10.1007/JHEP01(2016)061 [arXiv:1509.03499 [hep-ph]].

[18] B. Schenke, S. Schlichting and R. Venugopalan, Phys. Lett. B 747, 76 (2015) doi:10.1016/j.physletb.2015.05.051 [arXiv:1502.01331 [hep-ph]]. 